\title{
Phenotypic variation of tuberous sclerosis in a single extended kindred
}

Susan L Smalley, Frances Burger, Moyra Smith

\begin{abstract}
Tuberous sclerosis complex (TSC) is an autosomal dominant disorder with a great degree of phenotypic variability. Given the presence of two gene loci underlying this disorder, locus heterogeneity may account for some of the variability. However, significant within family variation suggests that different genes do not explain the majority of this variation. The purpose of this research is to identify physical and behavioural variation in expression of TSC in a single large extended kindred. TSC in this kindred is cosegregating with markers localised to chromosome 16p13.3. The expression of TSC in this kindred is quite variable with a substantial proportion of persons showing very mild physical expression of TSC. In contrast to very mild physical expression of TSC in some family members, there is a significant clustering of psychiatric disorders among persons affected with TSC compared to their unaffected relatives. This finding, coupled with the mild physical expression of TSC in some family members, supports a hypothesis that the TSC2 gene may present phenotypically as mild skin signs and significant behavioural problems.
\end{abstract}

(f Med Genet 1994;31:761-765)

Tuberous sclerosis complex (TSC) is an autosomal dominant disorder characterised by hamartomas and hamartias in many organs including brain, skin, heart, and kidney. ${ }^{1}$ There is a great degree of variation in clinical signs of TSC as reflected in the diversity of diagnostic criteria. ${ }^{12}$ Genetic heterogeneity is indicated from the localisation of TSC genes through linkage studies to two different chromosome locations, $9 \mathrm{q} 34$ and $16 \mathrm{p} 13.3$, with approximately equal proportions of families showing linkage to one of the two locations. ${ }^{3-5}$ Recently, the TSC2 gene was cloned. ${ }^{6}$ The gene, named tuberin, codes for a $5.5 \mathrm{~kb}$ transcript which is expressed in many cells including brain, kidney, skin, liver, adrenal gland, colon, and white blood cells. The predicted tuberin protein is 1784 amino acids with a calculated molecular mass of $198 \mathrm{kDa}$. There is a region of sequence homology of tuberin and GTPase activating protein (GAP) human GAP3 (or rap1GAP) as well as murine GAP.

Although locus heterogeneity may account for some of the clinical variation between families, there is, as yet, no evidence that phenotypic variation correlates strongly with locus heterogeneity based on families showing linkage to chromosome $9 v 16 .{ }^{78}$ Furthermore, this locus heterogeneity is not a viable explanation for observed within family variability of TSC which can be quite extensive. ${ }^{19}$ Alternative explanations for variable expressivity of TSC within families include non-inherited events (for example, somatic mutations), modifier genes, and dynamic genes (that is, unstable DNA), such as those identified in Huntington's disease, myotonic dystrophy, and fragile $\mathrm{X}$. Since there is no evidence of unstable trinucleotide repeats in the cloned TSC2 gene, this latter mechanism is an unlikely explanation for variability within chromosome 16 linked families.

In addition to the physical signs of TSC, neurological impairment has been recognised as a significant aspect of this disorder, reflected by the co-occurrence of mental retardation or seizures or both. Mental retardation (MR) occurs in approximately $50 \%$ of TSC persons while seizures are present in approximately $80 \%$ of TSC patients. ${ }^{1}$ Recently, there has been an increasing amount of research directed at identifying specific behavioural disorders among TSC affected patients. ${ }^{1011}$ In a review of 50 published case reports, we found that autism and autistic-like behaviour, hyperactivity/impulsivity, and aggression/ uncooperative behaviour occurred in approximately $36 \%, 26 \%$, and $48 \%$ of the sample. A majority of these persons had comorbid mental retardation (64\%) and seizures (90\%). In addition to these case reports, there have been epidemiological and clinic based studies of behavioural problems in TSC with autism occurring in these samples in approximately $25 \%$ of cases. ${ }^{1011}$ Recently, Gillberg et al ${ }^{12}$ reported very high rates of autism $(61 \%)$ and attention deficit disorder $(43 \%)$ in a Swedish population of 28 TSC persons. Although in that sample, 10/28 TSC patients did not have mental retardation, there remains a paucity of data directed at describing whether specific behavioural, psychiatric, or cognitive problems occur in non-retarded TSC populations.

In the present investigation, we report data regarding physical and behavioural expression of TSC in a large extended kindred consisting of two distantly related branches. The purpose of this research is two fold: (1) to examine phenotypic variability of TSC in a single extended kindred; (2) to determine whether specific behavioural or psychiatric disorders are increased in a sample of non-retarded TSC persons compared with their non-TSC affected relatives. 


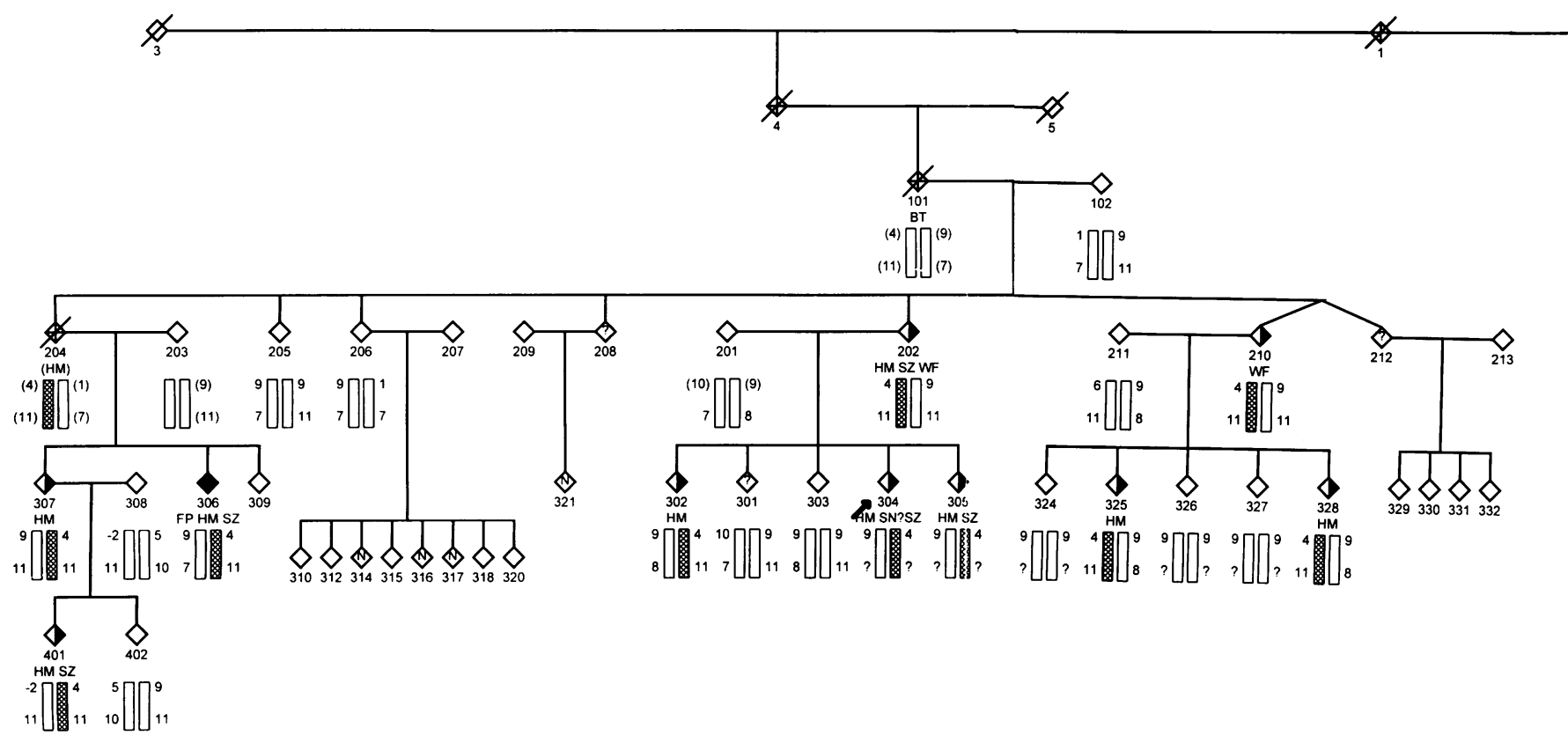

\section{Materials and methods SUBJECTS}

The sample consists of a large kindred with TSC shown in the figure. There are two branches in the kindred linked through a common male ancestor (subject 1). The family is composed of members of the Church of Jesus Christ of Latter-day Saints (LDS) located in Utah, thus extensive family histories, compiled by the Utah Genealogical Society, are maintained in the Utah Resource for Genetic and Epidemiology Research at the University of Utah. Based on these records, no other common ancestors have been identified for subjects $101,102,105,106$, or subjects $2-9$. The family was identified through subject 304 in the kindred. During our evaluations of this branch of the kindred, the second branch was identified through contact between the mother of 304 and the mother of 354 . This pedigree was not included in a previous report of linkage investigations in TSC families. ${ }^{13}$

\section{PHYSICAL EVALUATIONS}

Seventy-four persons have been evaluated by MS or a clinical geneticist or both using a Wood's light and brief eye evaluations under natural light conditions. Medical records were obtained for nine persons who had more extensive medical evaluations for TSC, including brain CT $(n=7)$ or MRI $(n=3)$ scans, skull $x$ rays (one subject), kidney ultrasounds (six subjects), echocardiograms (four subjects), and ophthalmological evaluations (two subjects). Physical findings for each family member based on the physical examination and medical records are noted on the pedigree. Among 74 subjects evaluated in a physical examination including Wood's light, 19 (10 in branch A, nine in branch B) meet suspected/possible $(n=$ 13) or definite $(n=6)$ criteria of TSC using the diagnostic criteria of Gomez. ${ }^{2}$ Five subjects were considered to have unknown status because they presented questionable skin signs, for example, a single hypomelanotic macule, a port wine stain, or confetti freckling on sun exposed areas only. Nine persons have not been evaluated as yet because they live out of state or were unavailable for physical evaluation at the times of testing.

Blood was drawn and permanent lymphoblastoid cell lines were established on 74 persons. Thirty-three subjects were typed for chromosome markers tightly linked to the TSC2 locus, D16S85 (HGM 11, 1991) or D16S291 (HGM 11, 1991) or both. D16S85 is a highly polymorphic VNTR detected using Southern blot analysis. ${ }^{14}$ D16S291 is a highly polymorphic dinucleotide repeat detected via PCR analysis following the methods discussed by Weber and May. ${ }^{15}$ Physical mapping of these markers suggests they are separated by approximately $3000 \mathrm{~kb}$ (personal communication, S Reeders, Yale University) and cloning of the TSC2 gene positions it between these two markers. ${ }^{6}$ In addition, persons were typed for $\mathrm{ABO}$ blood type, known to lie close to the TSC1 region on chromosome 9q34.3. Genotyping was conducted after phenotypic status was determined based on clinical evaluation.

\section{PSYCHIATRIC EVALUATIONS}

Psychiatric evaluations were completed on 37 subjects in the kindred by FB using the SADSLA for subjects 18 years of age and older ${ }^{16}$ or K-SADS-E for subjects 17 years of age and younger. ${ }^{17}$ These semi-structured interviews are widely used in genetic epidemiological research and have been shown to have good interrater reliability. ${ }^{1617}$ The Autism Diagnostic Interview (ADI) was administered to two mothers of three subjects in whom a possible diagnosis of autism or pervasive developmental disorder (PDD) was suggested from a family history interview. The ADI is an investigator based interview which has been shown to have excellent reliability in the diagnosis of autism. ${ }^{18}$ 


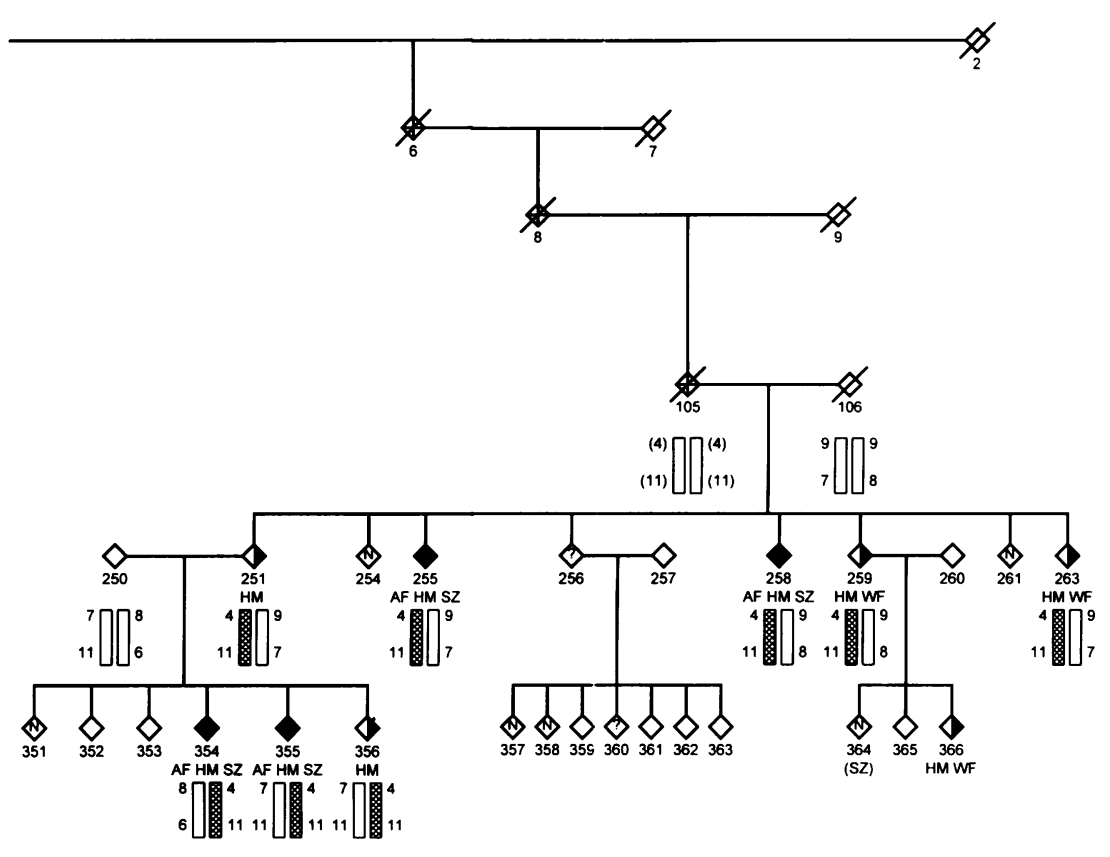

All interviews were videotaped and re-rated independently by an interviewer at UCLA trained in the administration of these semistructured interviews and blind to the subjects' TSC diagnosis. All cases with positive psychiatric diagnoses were subsequently reviewed by a diagnostic panel including trained family interviewers at UCLA and consulting psychiatrists, Dr Peter Tanguay or Dr James McCracken or both. Since the majority of TSC patients in this kindred are mildly affected, blind was maintained during direct interviews in the majority of cases and for all cases during the diagnostic panel review.

\section{DATA ANALYSES}

Two point lod scores were calculated using the ILINK package of LINKAGE $5.1^{19}$ on an IBM 486 computer. The genetic model for TSC used in the linkage analyses is autosomal dominant with the frequency of the TSC gene fixed at $1 / 20000$. Persons were considered affected with TSC if they met suspected, possible, or definite criteria ${ }^{1}$ based on physical examination. Penetrance for the TSC gene was fixed at 0.90 . Allele frequencies for D16S85 followed those described in HGM11 (1991). ${ }^{20}$ Allele frequencies for $\mathrm{ABO}$ were taken from Giblett. ${ }^{21}$ Allele frequencies for D16S291 were based on estimates obtained from a random sample of 100 persons in the CEPH pedigrees provided by Dr Pericak-Vance at Duke University (personal communication). Descriptive statistics, Student's $t$ tests, and $\chi^{2}$ tests, with Yates's adjustment for continuity, were calculated using SAS for Windows 6.08 (1993). ${ }^{22}$

\section{Results}

As shown in the figure, 19 persons met diagnostic criteria for TSC: six definite, 13 possible or suspected. Including all phenotype classifications as "affected", the maximum lod scores for TSC and chromosome 16p13.3 markers
Table 1 Maximum lod scores for TSC and chromosome $16 p 13.3$ markers

\begin{tabular}{lcc}
\hline Kindred & $\begin{array}{c}\text { D16S85 } \\
\text { lod }\end{array}$ & $\begin{array}{c}\text { D16S291 } \\
\text { lod }\end{array}$ \\
\hline Branch A & $4 \cdot 23$ & $0 \cdot 72$ \\
Branch B & $1 \cdot 71$ & $1 \cdot 83$ \\
Summed & $6 \cdot 20$ & $2 \cdot 81$ \\
Pooled* & $6 \cdot 86$ & 3.64 \\
\hline
\end{tabular}

All maximum lod scores were found at $\theta_{\mathrm{m}}=\theta_{\mathrm{f}}=0$.

${ }^{*}$ Kindred includes all connecting subjects with unknown marker status.

D16S85 and D16S291 are shown in table 1.

The maximum lod scores for each of these markers and TSC in this kindred is well over 3 , or 1000:1 odds of linkage (at $\theta=0$ ) for both markers when the kindred is treated as a whole. The maximum lod scores found for TSC and each marker, treating each branch as independent, are also consistent with linkage. As reflected on the pedigree, a haplotype consisting of alleles 4 (at D16S85) and 11 (at D16S291) is segregating with TSC in this kindred. Lod score values for $\mathrm{ABO}$ in this kindred are negative across all values of $\theta<0.50$ and linkage is excluded (lod $<-2.0$ ) up to $1.9 \mathrm{cM}$ from $\mathrm{ABO}$, a region spanning that thought to contain the TSC1 gene. ${ }^{23}$

Since many persons are mildly affected, we conducted a sensitivity analysis ${ }^{24}$ to evaluate the change in lod score when individual diagnostic status changed. Across all diagnostic changes (including affected, unaffected, and unknown) the lod scores varied from $5 \cdot 85$ to $7 \cdot 12$ for D16S85 and 2.05 to 3.72 for D16S291. The linkage findings were robust across various degrees of penetrance of the TSC gene (analyses not shown) and at a wide variety of marker genotype frequencies (analyses not shown).

\section{PSYCHIATRIC DIAGNOSES}

Among persons affected with TSC, there are four cases of mental retardation (IQ<70) based on age appropriate standardised tests. Two of these subjects were unable to complete a structured interview using the SADS-LA. One is institutionalised with a diagnosis of schizophrenia and the other lives semi-independently with no known psychiatric diagnosis. Among 37 pedigree members who completed both physical and psychiatric assessments, 17 are affected with TSC and 16 are not, based on physical criteria. However, as shown in table 2 , there is a significant clustering of psychiatric disorders among the TSC affected compared with non-TSC affected relatives in the kindred. While statistical comparisons are shown, the exact $p$ values may be somewhat different from those presented because the assumption of independent sampling is not met in the case of related persons.

Although there is dependency within and between these two groups because of the type of sampling (that is, a single kindred), the groups of affected and unaffected relatives were similar in degree of relationship. Coefficients of relationship (for example $\frac{1}{2}$ for sibs, $\frac{1}{4}$ for aunt-nephew, etc) were calculated for affected TSC members and unaffected TSC members based on a person's genetic relationship to 
Table 2 Frequency of psychiatric disorders among TSC affected and unaffected relatives

\begin{tabular}{|c|c|c|c|c|c|c|}
\hline \multirow[t]{2}{*}{ Psychiatric disorders } & \multicolumn{2}{|c|}{ TSC affected $(n=17)$} & \multicolumn{2}{|c|}{ TSC unaffected $(n=16)$} & \multirow[b]{2}{*}{$\chi^{2}, d f=1$} & \multirow[b]{2}{*}{$p$} \\
\hline & No & $\%$ & No & $\%$ & & \\
\hline Mood disorder & 6 & $35 \cdot 3$ & 1 & $6 \cdot 2$ & & \\
\hline Substance abuse & 1 & 5.9 & 0 & 0 & & \\
\hline Anxiety disorder & 10 & $58 \cdot 8$ & 2 & $12 \cdot 5$ & $5 \cdot 8$ & 0.016 \\
\hline $\begin{array}{l}\text { Attention deficit } \\
\text { Hyperactivity }\end{array}$ & 2 & $13 \cdot 3 *$ & 1 & $6.7^{*}$ & & \\
\hline Eating disorders & 2 & 11.8 & 0 & & & \\
\hline Any psychiatric disorder & 13 & $76 \cdot 5$ & 4 & $25 \cdot 0$ & $6 \cdot 8$ & 0.009 \\
\hline
\end{tabular}

person 304 (for those in branch A) or person 354 (for those in branch B). Since approximately equal numbers of affected and unaffected TSC members come from branch A and branch $B$, and since multiple generations separate the two branches, we estimated average coefficients of relationship in the two groups by pooling across branches. The average coefficient of relationship in the unaffected TSC relative group was 0.22 compared with 0.31 in the affected TSC group $(t=-1.33$, $\mathrm{df}=31, \mathrm{p}=0 \cdot 19$ ).

The types of psychiatric disorders observed in the two groups of relatives are shown in table 2 . As can be seen in the table, the increased rates of psychiatric disorders are primarily because of raised rates of anxiety disorders. There is a wide range of anxiety disorders seen in TSC affected subjects including panic disorder $(\mathrm{n}=$ 3) with and without agoraphobia, simple phobia $(n=5)$, obsessive-compulsive disorder $(n=$ $1)$, separation anxiety disorder $(\mathrm{n}=2)$, and overanxious disorder $(n=1)$. In addition, based on parental interview using the ADI, one subject meets ICD- $10^{25}$ criteria for PDD and one child meets ICD-10 criteria for autism using an ADI algorithm. Co-morbid diagnoses are present for both of these cases with the PDD child meeting DMSIIIR criteria for overanxious disorder and the child with autism also meeting criteria for depression NOS and attention deficit hyperactivity disorder. Using the ADOS for an observational assessment of these two children, they currently look very socially anxious and shy with developmental histories clearly PDD.

We compared severity of TSC in the two kindred branches for both physical and psychiatric problems. Based on 10 affected subjects in branch $A$ and nine affected subjects in branch $\mathrm{B}$, there is somewhat greater between than within phenotypic variation. In branch $B$, 4/9 subjects meet definite diagnostic criteria for TSC while only $1 / 10$ persons meet definite criteria in branch $\mathrm{A}$. The greatest physical difference is in the occurrence of facial angiofibromas in persons in branch $B$ (that is, 4/ 9) while none occur among branch A persons (Fisher's exact test, two tailed, $p=0.03$ ). There were no significant differences in the occurrence of psychiatric disorders or mental retardation between the two branches.

\section{Discussion}

In an extended kindred in Utah, we have found significant evidence that TSC cosegregates with markers localised to chromosome $16 \mathrm{p} 13.3$. Although classic presentation of TSC occurs in this kindred in six persons, the majority of those affected by TSC show very mild expression of TSC. Furthermore, for nine subjects who had brain MRI or CT scans or both, no cortical tubers or subependymal nodules characteristic of TSC were present; these subjects have only hypopigmented macules or confetti-like freckling. Given the strong evidence of linkage and the presence of four persons with pathognomonic TSC signs, the data suggest that the TSC2 gene is segregating in this kindred and expressed very mildly. In addition to mild physical expression, there is substantial evidence that the TSC2 gene is expressed behaviourally. We found significantly raised rates of psychiatric disorders, particularly anxiety disorders among TSC gene carriers in this kindred. Although behavioural problems have been noted in TSC populations, this is the first report, to our knowledge, suggesting that a certain psychiatric disorder (for example, anxiety) may be increased among those affected by TSC without co-morbid mental retardation. Furthermore, our finding of developmental histories consistent with autism/PDD in two subjects in the kindred who also have co-morbid anxiety/depression suggests that autisticlike behaviour in non-retarded TSC persons may be, in part, a reflection of extreme shyness or social anxiety.

There is substantial phenotypic variability in the kindred and these preliminary data suggest that the variation between two branches of the kindred is somewhat greater than that found within each kindred. Since the more severely affected branch is not the one through which the family was identified, it is unlikely that this finding is a result of ascertainment. Although this finding is suggestive of a genetic mechanism underlying the phenotypic variability of TSC, for example, a modifier gene or genes, it is based on very small sample sizes. We hope to identify additional family members related to this kindred through the common ancestor and to test this hypothesis further in the future.

These findings are limited, at present, because they are based on a single large kindred segregating TSC2. Since not all family members have, as yet, been evaluated, there may be a bias towards TSC affected members more often being assessed and having psychopathology. However, three aspects of the present sample suggest this is not a likely explanation for the current findings. First, approximately equal numbers of affected $(n=$ 17) and unaffected $(n=16)$ have completed psychiatric evaluations and equal proportions come from branch A and branch B. Second, affected family members are similar to unaffected relatives in degree of genetic relatedness suggesting the findings are not a function of greater genetic similarity in one group. Third, a majority of affected subjects in the study were unaware of their TSC affected status before the onset of the study; hence it is unlikely that affected status biased participation in the study. 
In conclusion, these findings support the hypothesis that TSC, specifically TSC2 (localised to chromosome $16 \mathrm{p} 13.3$ ), may be expressed very mildly, merely by the presence of hypomelanotic macules or confetti-like freckling. Furthermore, at least in this kindred, there is a significant clustering of anxiety disorders in these mildly affected TSC subjects compared with their non-TSC relatives. Since there are data supporting the hypothesis that the TSC2 gene functions as a tumour suppressor gene ${ }^{26}$ these findings are intriguing for several reasons. First, it raises the possibility that TSC gene expression is modified by other genes. A genetic modifier may be present which interacts with the tuberin protein on TSC2 and modifies the likelihood of a second hit occurring. Under this model, branch A and branch B may differ in the occurrence of this modifier. Second, it raises the possibility that the presence of a single TSC2 gene, in the absence of a second hit, may lead to abnormal cell migration or differentiation in different organs, including the brain, which are not detected or classified as hamartomata. An example of this would be the absence of cortical tubers or subependymal nodules on MRI but the presence of, for example, grey matter heterotopias or cytoarchitectural abnormalities detected by volumetric differences. Such brain tissue involvement, while not pathognomonic of TSC, may underlie specific behavioural, cognitive, and psychiatric sequelae of this condition. Unpublished data collected by our group suggest that significant volumetric brain differences (in cerebellar vermis) occur in TSC persons with autism compared to TSC persons without autism which lends support to this hypothesis. Such cytoarchitectural abnormalities could lead to dysregulation of a specific neurotransmitter system, for example, $\lambda$-aminobutyric acid (GABA) activity, a major CNS inhibitory transmitter that has been found to play a role in both seizures and anxiety disorders. ${ }^{27}$ Whether such a mechanism accounts for the specific behavioural sequelae seen in this kindred would require morphological and functional analyses of putative brain regions using MRI volumetric assessments and other methodologies such as PET or NMR spectroscopy.

The present findings suggest that a specific behavioural phenotype may occur in TSC2 gene carriers without mental retardation including anxiety disorders and perhaps autism/ PDD. These findings have important implications for clinical evaluations of TSC gene carriers including assessment and treatment for behavioural and psychiatric difficulties. Furthermore, a routine Wood's lamp examination in subjects with severe anxiety disorders or autism or PDD may identify TSC as an underlying mechanism in a small percentage of cases.
This work was supported in part by NIH grants RO1MH44742 and 2R01HD23745 and funding from NTSA and the Villicana Family Trust. Partial support for the Utah Population Database (UPDB) is provided by 2 P30 CA-42014-04. We thank especially the family members participating in this research; KayMegan Washington, James McCracken, Peter Tanguay, and Robert Asarnow for their help in diagnostic classification; Elizabeth Chambers for her help in data collection; Julie Russell zabeth Chambers for her help in data collection; Julie Russell and Griselda Gutierrez for manuscript preparation, data entry, and checking; Kathy Handa for her excellent technical

1 Gomez MR. Tuberous sclerosis. 2nd ed. New York: Raven Press, 1988.

2 Gomez MR. Phenotypes of the tuberous sclerosis complex with a revision of diagnostic criteria. In: Johnson WG, Gomez MR, eds. Tuberous sclemsis and allied disorders. Ann $N Y$ Acad Sci 1991;615:1-7.

3 Connor JM, Pirrit LA, Yates JR, et al. Linkage of the tuberous sclerosis locus to a DNA polymorphism detected by v-abl. $₹$ Med Genet $1987 ; 24: 544-6$.

4 Northrup H, Kwiatkowski DJ, Roach ES, et al. Evidence for genetic heterogeneity in tuberous sclerosis: one locus for genetic heterogeneity in tuberous sclerosis: one locus f Hum Genet 1992;51:709-20.

5 Kandt RS, Haines JL, Smith M, et al. Linkage of an important gene locus for tuberous sclerosis to a chromosome 16 marker for polycystic kidney disease. Nature Genet 1992;2:37-41.

6 European Chromosome 16 Tuberous Sclerosis Consortium. Identification and characterization of the tuberous sclerosis gene on chromosome 16. Cell 1993;75:1305-15.

7 Northrup H, Wheless JW, Bertin TK, Lewis RA. Variability of expression in tuberous sclerosis. $\mathcal{F}$ Med Genet 1993;30: 41-3.

8 Winship IM, Connor JM, Beighton PH. Genetic heterogeneity in tuberous sclerosis: phenotypic correlations. erogeneity in tuberous sclerosis:

9 Nevin NC, Pearce WG. Diagnostic and genetical aspects of tuberous sclerosis. 7 Med Genet 1968;5:273-80.

10 Hunt A, Shepherd C. A prevalence study of autism in tuberous sclerosis. $\mathcal{F}$ Autism Dev Discord 1993;23:323-39.

11 Smalley SL, Tanguay PE, Smith M, Gutierrez G. Autism and tuberous sclerosis. F Autism Dev Discord 1992;22 $339-55$

12 Gillberg IC, Gillberg C, Ahlsen G. Autistic behaviour and attention deficits in tuberous sclerosis: a population-based study. Dev Med Child Neurol 1994;36:50-6.

13 Smith M, Smalley SL, Cantor R, et al. Mapping of a gene determining tuberous sclerosis to human chromosome 11q14-11q23. Genomics 1990;6:105-14.

14 Jarman AP, Nicholls RD, Weatherall DJ, et al. Molecular characterization of a hypervariable region downstream of the human-alpha-globin gene cluster. $E M B O$ f $1986 ; 5$ : $1857-63$.

15 Weber JL, May PE. Abundant class of human DNA polymorphisms which can be typed using the polymerase chain reaction. Am f Hum Genet 1989;44:388-96.

16 Mannuzza S, Fyer AJ, Klein DF, Endicott J. Schedule for affective disorders and schizophrenia - lifetime version modified ive disorders and schizophrenia - lifetime version modified

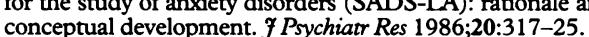

17 Weissman MM, Wickramaratne P, Warner V, et al. Assessing psychiatric disorders in children: discrepancies between mothers' and children's reports. Arch Gen Psychiatry 1987 44:747-53.

18 Le Couteur A, Rutter M, Lord C, et al. Autism diagnostic interview: a standardized investigator-based instrument. $f$ Autism Dev Disond 1989;19:363-87.

19 Ott J. Linkage analysis package II: users' guide to analysis programs. Version 5.1 for IBM PC/compatibles, Columbi University, 1992.

20 Solomon E, Rawlings C, eds. Eleventh international workshop on human gene mapping. Cytogenet Cell Genet 1991; 58: $1-4$.

21 Giblett ER. Genetic markers in human blood. Oxford: Blackwell, 1969.

22 SAS Procedures guide. Version 6. Cary, North Carolina: SAS Institute Inc, 1993.

23 Nellist M, Brooke-Carter PT, Connor JM, et al. Identification of markers flanking the tuberous sclerosis locus on chromosome 9 (TSC1). $\Im$ Med Genet 1993;30:224-7.

24 Hodge SE, Greenburg DA. Sensitivity of lod scores to changes in diagnostic status. Am ₹ Hum Genet 1992;50: 1053-66.

25 World Health Organization. ICD-10 1992 draft of chapter 5 categories F00-F99. Mental behavioural and developmental disorders. Geneva: WHO, 1992.

26 Smith M, Handa K, Wei H, Spear G. Loss of heterozygosity for chromosome $16 \mathrm{p} 13.3$ markers in renal hamartoma from tuberous sclerosis patients. Poster presented at the 43rd Annual Meeting, New Orleans, Louisiana. $A m f$ Hum Genet Suppl 1993;53:366.

27 Kaplan HI, Sadock BJ, eds. Synopsis of psychiatry: behavioral sciences clinical psychiatry. 6 th ed. Baltimore: Williams and Wilkins, 1991. 